\title{
Universiteit
}

Leiden

The Netherlands

\section{Ultrafast dynamics of correlation bands following XUV molecular photoionization}

Hervé, M.; Despré, V.; Castellanos Nash, P.; Loriot, V.; Boyer, A.; Scognamiglio, A.; ... ; Lépine, F.

\section{Citation}

Hervé, M., Despré, V., Castellanos Nash, P., Loriot, V., Boyer, A., Scognamiglio, A., ... Lépine, F. (2020). Ultrafast dynamics of correlation bands following XUV molecular photoionization. Nature Physics, 17(3), 327-331. doi:10.1038/s41567-020-01073-3

Version:

Publisher's Version

License:

Licensed under Article 25fa Copyright Act/Law (Amendment Taverne)

Downloaded from: https://hdl.handle.net/1887/3276753

Note: To cite this publication please use the final published version (if applicable). 


\title{
Ultrafast dynamics of correlation bands following XUV molecular photoionization
}

\author{
M. Hervé ${ }^{1,4}$, V. Despré2, , P. Castellanos Nash ${ }^{3}$, V. Loriot ${ }^{1}$, A. Boyer ${ }^{1}$, A. Scognamiglio', G. Karras1, \\ R. Brédy $\oplus^{1}$, E. Constant ${ }^{1}{ }^{1}$, A. G. G. M. Tielens ${ }^{3}$, A. I. Kuleff $\odot^{2}$ and F. Lépine $\circledast^{1 凶}$
}

\begin{abstract}
Modern ultrashort X-ray/XUV (extreme ultraviolet) sources provide unique opportunities to investigate the primary reactions of matter upon energetic excitation. Understanding these processes in molecules on ultrafast timescales is required to improve bespoke high-energy radiation detectors, nanomedicine schemes or to study the molecular composition of interstellar media. However, current experiments struggle to provide a general framework because of the uniqueness and complexity of each system. Here we show the universal role of correlation bands-features created by electron correlation. This is done by studying ultrafast energy relaxation of size-scalable two-dimensional molecules following ionization by an ultrashort XUV pulse. We observed long lifetimes that nonlinearly increase with the number of valence electrons. A general law based on solid-like electron-phonon scattering is proposed, which explains both our results and previously reported measurements. This offers new opportunities in attosecond science and high-energy photophysics.
\end{abstract}

Energetic irradiation of molecular-sized structures followed by ultrafast charge and energy flow is a general phenomenon encountered in nature and technology. For example, the detection of energetic radiations in organic scintillators relies on detector devices incorporating molecules such as polycyclic aromatic hydrocarbons (PAHs), which convert high-energy radiation into low-energy light emission $^{1}$. In nanomedicine, the high energy deposited in nanosized structures can be used to enhance cancer treatments ${ }^{2}$. It also plays a crucial role in space, where molecules evolve in interstellar media upon interaction with energetic photons, shaping molecular structures and eventually leading to the appearance of prebiotic molecules as a first step in the emergence of life in the Universe on astrophysical timescales ${ }^{3}$.

Ultrafast extreme ultraviolet (XUV) technology has opened new opportunities in these contexts by providing tools such as ultrashort vacuum ultraviolet (VUV), XUV or X-ray pulses, allowing us to decipher the initiated dynamics, with the ultimate aim of observing and controlling photoinduced reactions on the femtosecond ${ }^{4-6}$ and even attosecond ${ }^{7,8}$ timescales. Since the early days of attosecond science, ground-breaking results have been obtained by imaging ultrafast molecular transformations using attosecond pulses, soon followed by the possibility to control electron localization on the attosecond timescale in $\mathrm{H}_{2}$ (ref. ${ }^{9}$ ) and in small molecules ${ }^{10,11}$. However, larger molecular species have been investigated far less because of the experimental and theoretical challenges. Remarkable results were obtained in phenylalanine $e^{12}$, where oscillating features were shown on the timescale of a few femtoseconds that were attributed to attosecond hole migration ${ }^{13,14}$ through the molecular backbone, soon confirmed by similar investigations on tryptophan ${ }^{15}$.

These experiments have demonstrated the existence of new exciting processes that could be understood in terms of 'pure' electron dynamics ${ }^{16}$ yet questioning the role played by other degrees of freedom ${ }^{17-23}$. However, the lack of an adequate many-body quantum theory and the limited available experimental data have restricted our understanding of the underlying electron-vibration energy transfer mechanisms.

We performed a systematic investigation of XUV-induced relaxation in size-scalable two-dimensional (2D) molecular systems-PAHs. Our results reveal an unexpected simple trend for the size-dependent relaxation lifetime that provides a general scaling law. The process is understood as dynamics in the so-called correlation bands ${ }^{24}$, which are intrinsic features of the high-energy photoionization of many-electron systems, created by the electron correlation. An electron-phonon scattering model is proposed as a tool to describe dynamics in correlation bands in any complex molecule. We show that it provides a general framework to understand XUV-induced processes in currently reported experiments.

The general concept of the experiment is presented in Fig. 1a. Molecules are irradiated by an ultrashort 20 -fs XUV pulse (centred around $23 \mathrm{eV}$ ), leading to ionization of inner-valence shells. This produces excited cationic molecules, which are probed by a second ionization step induced by a delayed 25 -fs 800 -nm pulse, leading to doubly charged, stable cations detected by a time-of-flight spectrometer (TOF). The variation of the dication yield as a function the XUV-infrared (XUV-IR) pump-probe delay provides direct information on the non-adiabatic relaxation of the excited states populated below the ionization threshold of the dication (Supplementary Information).

The XUV-induced dynamics was measured for a series of seven planar (2D) PAH molecules (from naphthalene $\left(\mathrm{C}_{10} \mathrm{H}_{8}\right)$, which contains two aromatic rings, to hexabenzocoronene $\left(\mathrm{C}_{42} \mathrm{H}_{18}, \mathrm{HBC}\right)$, built from 13 rings). The results obtained for coronene $\left(\mathrm{C}_{24} \mathrm{H}_{12}\right)$ are shown in Fig. 1b; these correspond to a fast increase of the yield around zero delay followed by an exponential decay of a few tens of femtoseconds. A single time constant is sufficient to represent the dynamics, which, in the case of coronene, is $51 \pm 7 \mathrm{fs}$. Varying the infrared intensity and XUV spectrum (Fig. 1c) changes neither the observed behaviour nor the time constant, but only the signal-to-noise ratio. Therefore, we conclude that the measured dynamics is intrinsic to the excited cationic states created below the double ionization threshold, because of the non-resonant character of the photoionization process.

'Univ Lyon, Univ Claude Bernard Lyon 1, CNRS, Institut Lumière Matière, F-69622, Villeurbanne, France. ${ }^{2}$ Theoretische Chemie, PCl, Universität Heidelberg, Heidelberg, Germany. ${ }^{3}$ Leiden Observatory, Leiden University, Leiden, The Netherlands. ${ }^{4}$ These authors contributed equally: M. Hervé, V. Despré.

凶e-mail: franck.lepine@univ-lyon1.fr 
$\mathbf{a}$
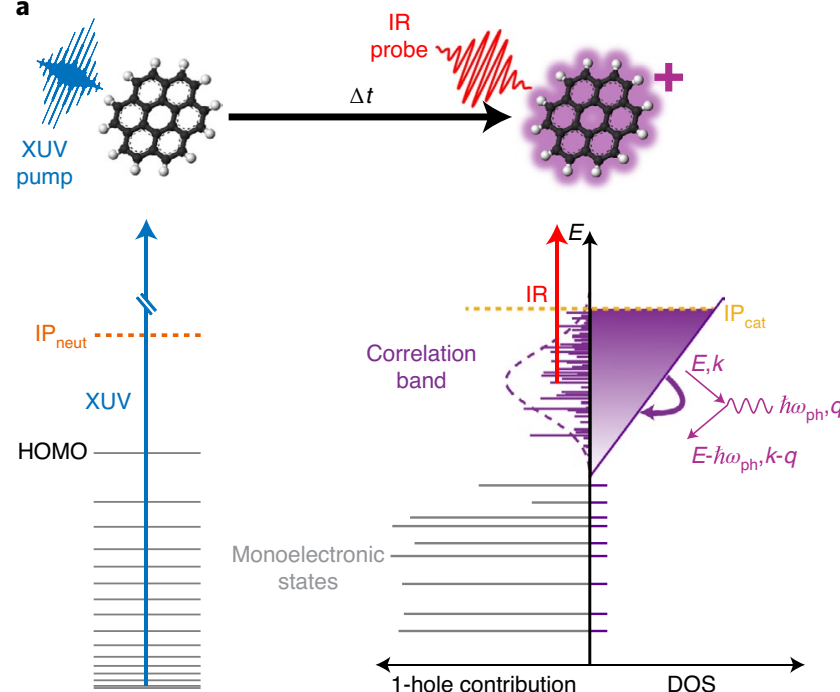

b
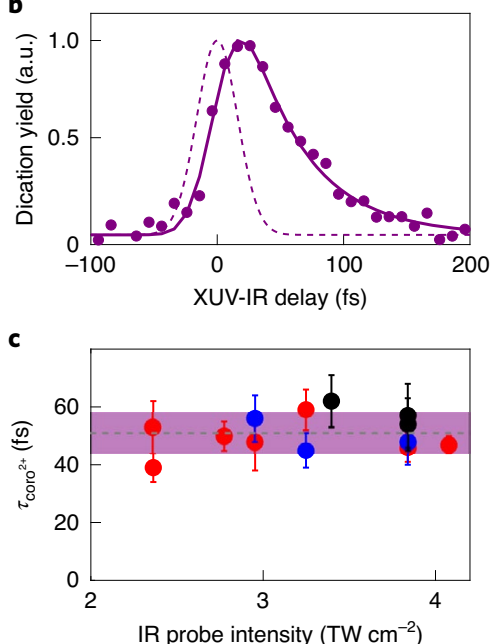

Fig. 1 | Dynamics in correlation bands. a, lonization of the inner-valence shells of molecules, well below the highest occupied molecular orbital (HOMO), by an XUV pump pulse (left) leads to the formation of a correlation band (CB) composed of a multitude of strongly coupled multielectronic states below the double ionization threshold, $\mathrm{IP}_{\text {cat }}$ (right). The CB in PAHs can then be described as a solid-like band with a linear density of state (DOS). The subsequent relaxation occurs through electron-phonon scattering and is probed by ionization with the delayed infrared pulse, creating a stable dication. b, Measured dication yield as a function of the XUV-IR delay for coronene (purple dots), together with the exponential fit (purple full line), and the cross-correlation between the pump and the probe pulses (dashed line). c, Measured relaxation time in coronene as a function of the probe intensity, for different XUV spectra (see Supplementary Fig. 8 for the corresponding spectra; central energies: blue =22.3 eV, red =24.1 eV, black =28.7 eV).

The associated error bars correspond to the standard deviation of the fitting procedure for each measurement.

The relaxation dynamics follows a similar exponential trend for all measured molecules. The decay timescales are presented in Fig. $2 \mathrm{j}$ for all the molecules as a function of the number of valence electrons, from benzene ${ }^{21}$ to HBC. We clearly observe a smooth increase of the decay time as a function of the number of valence electrons. We emphasize that one needs to study a set of PAHs, including large ones, to exhibit the nonlinear evolution of the decay time. It also allows us to provide a general insight into the XUV-induced dynamics.

The removal of an electron with an XUV photon can leave the molecules in any of a large number of excited states. For high enough photon energy (typically above $10 \mathrm{eV}$ ), the character of these excited states changes qualitatively from monoelectronic (corresponding to one-electron transitions) to multielectronic (describing correlated transitions of more than one electron). As discussed by Cederbaum et al. ${ }^{25}$, typically above $15 \mathrm{eV}$, the representation in terms of molecular orbitals eventually breaks down completely. The removal of a single electron populates many multielectronic states, whose number and density rapidly grow, forming structures called 'correlation bands' (CBs). These CBs resemble the bands encountered in solid matter (Fig. 1a, right) and lie below the double ionization threshold (typically at $\sim 20 \mathrm{eV}$ ). Despite their generality, the dynamics in these CBs has never been experimentally studied elsewhere. Thus, we propose a general model based on an analogy with solid-state physics ${ }^{26,27}$ and show that they play an essential role in XUV-induced dynamics in general.

In contrast to the molecular view, where the electronic states are typically non-adiabatically coupled pairwise, the states constituting these bands are all strongly coupled to each other ${ }^{28,29}$ (Supplementary Information). Consequently, the dynamics in CBs can be seen as an electron-phonon scattering process, transferring energy from the electronic band to the phonons. Based on general considerations on electron correlation, and a linear increase of the density of states (DOS) as a function of energy, a general expression for the relaxation lifetime can be derived (see Supplementary Information for full derivation):

$$
\tau=\tau_{0} \ln \frac{N}{N_{\mathrm{CB}}}
$$

with

$$
\tau_{0}=\frac{3 \hbar}{2 \pi g_{0}^{2} \omega_{0} \alpha}
$$

where $g_{0}$ is the mean electron-phonon coupling strength, $\omega_{0}$ is the mean phonon energy and $\alpha$ is the slope of the DOS as a function of energy. Interestingly, equation (1) depends only on two parameters $\left(\tau_{0}, N_{\mathrm{CB}}\right)$, which are determined by the fundamental properties of the corresponding molecule. In our model, $\tau_{0}$ can be interpreted as the characteristic time of the electron-phonon scattering process and $N_{\mathrm{CB}}$ as the minimum number of electrons where the phenomenon begins. The latter depends on the strength of the electron correlation: in strongly correlated systems, $N_{\mathrm{CB}}$ can be small, while in weakly correlated ones, it will be large. The relaxation lifetime $\tau$ corresponds to the timescale for electronic energy loss, driving a single exponential decay.

Although equation (1) is derived for a specific molecule, a fit of the overall PAH dataset with a single set of free parameters $\left(\tau_{0}, N_{\mathrm{CB}}\right)$ shows very good agreement (with an accuracy of $r^{2}=97 \%$, (purple curve, Fig. 2j), much better than, for example, a linear dependence $\left(r^{2}=88 \%\right.$, not shown)), yielding $\tau_{0}=24 \pm 3 \mathrm{fs}$ and $N_{\mathrm{CB}}=14 \pm 3$. This shows that the constants $\left(\tau_{0}, N_{\mathrm{CB}}\right)$ carry information not only on a specific molecule, but also on the intrinsic properties of the whole family of PAH molecules. For a given molecular family, the strength of the electron correlation is not expected to vary strongly, therefore $N_{\mathrm{CB}}$ will vary slowly with size.

To obtain a deeper insight into these results, we performed ab initio calculations. The DOS was computed using multielectronic theory 

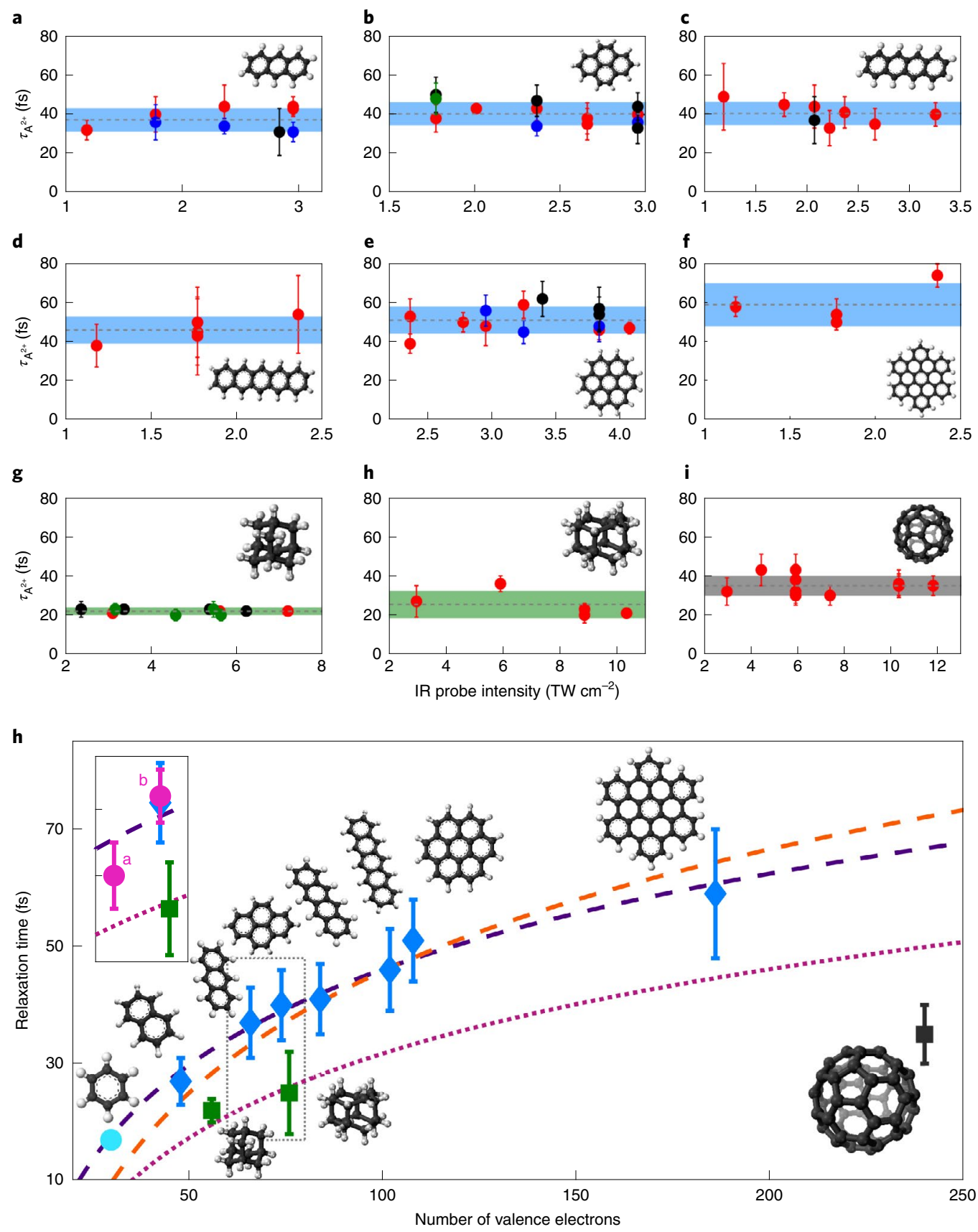

Fig. 2 | Scaling law in the relaxation of CBs for 2D and 3D structures. a-i, Measured decay for all the different PAHs and 3D systems as a function of IR intensity and the XUV spectrum (see Supplementary Fig. 8 for the corresponding spectra; central energies: blue $=22.3 \mathrm{eV}, \mathrm{red}=24.1 \mathrm{eV}$, black $=28.7 \mathrm{eV}$, green $=33.8 \mathrm{eV}$ ), with associated error bars: anthracene $(\mathbf{a})$, pyrene $(\mathbf{b})$, tetracene $(\mathbf{c})$, pentacene $(\mathbf{d})$, coronene $(\mathbf{e}), \mathrm{HBC}(\mathbf{f})$, adamantane $(\mathbf{g})$, diamantane (h) and $\mathrm{C}_{60}(\mathbf{i}) . \mathbf{j}$, The relaxation times, reported as a function of the number of valence electrons, for PAHs (blue diamonds) and 3D structures (green and black squares), together with their error bars, taken as the standard deviation of all the measurements for each molecule. The orange dashed line and pink dotted line correspond to equation (1), using parameters determined by ab initio calculations, while the purple dashed curve corresponds to a direct fit of the PAH lifetimes with equation (1), with free parameters (see main text). The lifetime for benzene ${ }^{21}$ is also shown (light blue circle). Inset: a zoomed view of the area outlined with a grey rectangle, where previously reported data are also included (magenta circles): phenylalanine $\left(\mathrm{C}_{9} \mathrm{H}_{11} \mathrm{NO}_{2}\right.$, label a) ${ }^{12}$ and caffeine $\left(\mathrm{C}_{8} \mathrm{H}_{10} \mathrm{~N}_{4} \mathrm{O}_{2}\right.$, label b) ${ }^{30}$.

in the case of naphthalene, anthracene and pyrene (Fig. 3), validating the linearity of the DOS near the onset of the CB, even for small molecules (states in purple, Fig. 3). Quantum non-adiabatic relaxation calculations performed on naphthalene ${ }^{28}$ were used to compute the two parameters (Supplementary Information). We would like to stress that only a limited number of active vibrational modes need to be considered for the determination of $\omega_{0}$. This reduction of dimensionality is important for future theoretical studies. We obtained $\tau_{0}^{\text {theo }}=30 \mathrm{fs}$ and $N_{\mathrm{CB}}^{\text {theo }}=22$, in good agreement with the result of the fit (orange dashed line, Fig. $2 \mathrm{j}$ ). This corroborates the relation between non-adiabatic molecular relaxation, computed with ab initio many-body molecular theories, and the electronphonon scattering defining our model. Although accurate ab initio calculations can be used to estimate the parameters for small 

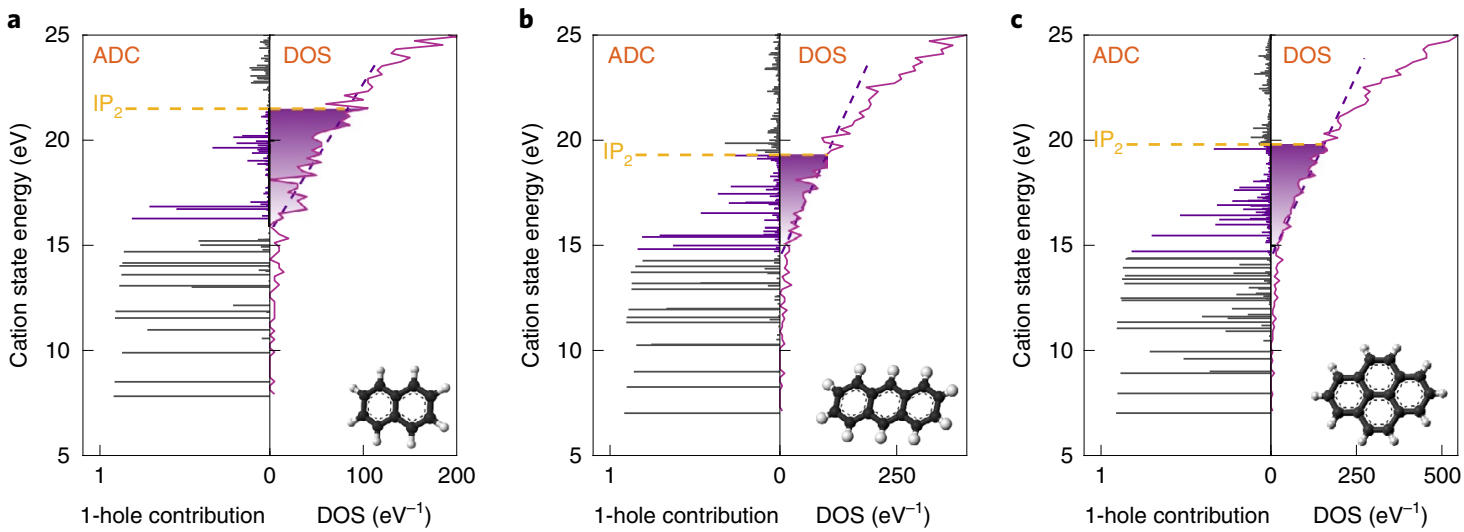

Fig. 3 | Analogy between CBs and solid-like band structures. a, Third-order algebraic diagrammatic construction (ADC(3)) calculations of the cationic states in naphthalene (left). The length of the line corresponds to the one-hole contribution to the state. Because the states are normalized to 1, the shorter the line, the stronger the multielectronic character. Above $15 \mathrm{eV}$, the breakdown of the molecular orbital picture leads to the creation of a CB. The DOS is extracted from these calculations and plotted as a function of energy (shown on the right). A linear increase of the DOS up to the second ionization potential is observed, fitted by the purple dashed line. $\mathbf{b}, \mathbf{c}$, Same analysis as in a for anthracene (b) and pyrene (c).

molecules, the model allows us to extract precise information on the relaxation dynamics, even for very large systems. Once calibrated on a few systems, our model can predict the behaviour of any molecule of the family.

Consequently, a given set of parameters determines the behaviour of a given class of molecules according to their CB. This idea has been tested by measuring different types of system, following the same experimental procedure (green and black squares, Fig. 2j). We see that diamondoids and fullerenes do not follow the PAH curve and new sets of parameters have to be determined for these structures. We determined a $\tau_{0}^{\text {Dia }}=21 \mathrm{fs}$ for diamondoids (Supplementary Information), and assuming that $N_{\mathrm{CB}}^{\mathrm{Dia}}=N_{\mathrm{CB}}^{\mathrm{PAH}}$, we obtain a new scaling law that agrees well with the measured diamondoid data (dotted pink curve, Fig. 2j). Interestingly, the lifetime observed in $\mathrm{C}_{60}$ lies clearly off the diamondoid curve, showing that $\mathrm{C}_{60}$ belongs to a different molecular family and should thus follow a different scaling law.

CBs appear due to the universal breakdown of the molecular orbital picture and are present in any complex-enough molecule. The dynamics in CBs is driven by electron-phonon scattering, which is also ubiquitous. Therefore, the process should arise in any larger molecular system, and might already explain previous results obtained in biomolecules ${ }^{12,30}$ (inset, Fig. 2j). It is indeed very striking that pyrene and caffeine, which have exactly the same number of valence electrons (74) but very different molecular properties, give exactly the same decay timescale ( $40 \mathrm{fs}$ ). This means that their $\mathrm{CBs}$ have the same properties. On the other hand, phenylalanine lies between the PAH and diamondoid curves and therefore could correspond to a third class of $\mathrm{CB}$. One of the remarkable features of the $\mathrm{CBs}$ is that the excited population is 'trapped' there for an unexpectedly long time. Recent experiments have shown that electronic ${ }^{12}$ and nuclear ${ }^{28}$ coherences could survive a few tens of femtoseconds, while calculations based on few coupled electronic states ${ }^{17,23}$ predict a much faster dephasing. We presume that high-density CBs might be responsible for the long-lived coherences observed in these experiments, showing the importance of many-body quantum effects.

Our findings also bring important information for molecular astrophysics, as the dynamics in CBs might strongly influence the competition between ionization, direct dissociation and radiative channels $^{31}$. The relaxation-time evolution law can also be incorporated easily into the astrochemistry models used for simulating XUV-induced chemistry in interstellar media.

\section{Online content}

Any methods, additional references, Nature Research reporting summaries, source data, extended data, supplementary information, acknowledgements, peer review information; details of author contributions and competing interests; and statements of data and code availability are available at https://doi.org/10.1038/ s41567-020-01073-3.

Received: 23 March 2020; Accepted: 23 September 2020; Published online: 16 November 2020

\section{References}

1. Maddalena, F. et al. Inorganic, organic and perovskite halides with nanotechnology for high-light yield X- and $\gamma$-ray scintillators. Crystals $\mathbf{9}$, 88 (2019).

2. Chatterjee, D. K., Fong, L. S. \& Zhang, Y. Nanoparticles in photodynamic therapy: an emerging paradigm. Adv. Drug Deliv. Rev. 60, 1627-1637 (2008).

3. Tielens, A. G. G. M. Interstellar polycyclic aromatic hydrocarbon molecules. Annu. Rev. Astron. Astrophys. 46, 289-337 (2008).

4. Attar, A. R. et al. Femtosecond X-ray spectroscopy of an electrocyclic ring-opening reaction. Science 356, 54-59 (2017).

5. Berrah, N. et al. Femtosecond-resolved observation of the fragmentation of buckminsterfullerene following X-ray multiphoton ionization. Nat. Phys. 15, 1279-1283 (2019).

6. Pertot, Y. et al. Time-resolved X-ray absorption spectroscopy with a water window high-harmonic source. Science 355, 264-267 (2017).

7. Nisoli, M., Decleva, P., Calegari, F., Palacios, A. \& Martín, F. Attosecond electron dynamics in molecules. Chem. Rev. 117, 10760-10825 (2017).

8. Kobayashi, Y., Chang, K. F., Zeng, T., Neumark, D. M. \& Leone, S. R. Direct mapping of curve-crossing dynamics in IBr by attosecond transient absorption spectroscopy. Science 365, 79-83 (2019).

9. Sansone, G. et al. Electron localization following attosecond molecular photoionization. Nature 465, 763-766 (2010).

10. Neidel, C. H. et al. Probing time-dependent molecular dipoles on the attosecond time scale. Phys. Rev. Lett. 111, 033001 (2013).

11. Drescher, L. et al. State-resolved probing of attosecond timescale molecular dipoles. J. Phys. Chem. Lett. 10, 265-269 (2019).

12. Calegari, F. et al. Ultrafast electron dynamics in phenylalanine initiated by attosecond pulses. Science 346, 336-339 (2014).

13. Cederbaum, L. S. \& Zobeley, J. Ultrafast charge migration by electron correlation. Chem. Phys. Lett. 307, 205-210 (1999).

14. Kuleff, A. I. \& Cederbaum, L. S. Ultrafast correlation-driven electron dynamics. J. Phys. B 47, 124002 (2014).

15. Lara-Astiaso, M. et al. Attosecond pump-probe spectroscopy of charge dynamics in tryptophan. J. Phys. Chem. Lett. 9, 4570-4577 (2018).

16. Lépine, F., Ivanov, M. Y. \& Vrakking, M. J. J. Attosecond molecular dynamics fact or fiction? Nat. Photon. 8, 195-204 (2014).

17. Vacher, M., Bearpark, M. J., Robb, M. A. \& Malhado, J. P. Electron dynamics upon ionization of polyatomic molecules: coupling to quantum nuclear motion and decoherence. Phys. Rev. Lett. 118, 083001 (2017). 
18. Despré, V., Golubev, N. V. \& Kuleff, A. I. Charge migration in propiolic acid: a full quantum dynamical study. Phys. Rev. Lett. 121, 203002 (2018).

19. Belshaw, L. et al. Observation of ultrafast charge migration in an amino acid. J. Phys. Chem. Lett. 3, 3751-3754 (2012).

20. Månsson, E. P. et al. Ultrafast dynamics in the DNA building blocks thymidine and thymine initiated by ionizing radiation. Phys. Chem. Chem. Phys. 19, 19815-19821 (2017).

21. Galbraith, M. C. E. et al. XUV-induced reactions in benzene on sub-10-fs timescale: nonadiabatic relaxation and proton migration. Phys. Chem. Chem. Phys. 19, 19822-19828 (2017).

22. Curchod, B. F. E. \& Martínez, T. J. Ab initio nonadiabatic quantum molecular dynamics. Chem. Rev. 118, 3305-3336 (2018).

23. Arnold, C., Vendrell, O., Welsch, R. \& Santra, R. Control of nuclear dynamics through conical intersections and electronic coherences. Phys. Rev. Lett. 120, 123001 (2018).

24. Deleuze, M. S. \& Cederbaum, L. S. Formation of satellite bands in the ionization spectra of extended systems. Phys. Rev. B 53, 13326 (1996).

25. Cederbaum, L. S., Domcke, W., Schirmer, J. \& von Niessen, W. Correlation effects in the ionization of molecules: breakdown of the molecular orbital picture. Adv. Chem. Phys. 65, 115-159 (1986).
26. Tielrooij, K. J. et al. Photoexcitation cascade and multiple hot-carrier generation in graphene. Nat. Phys. 9, 248-252 (2013).

27. Song, J. C. W., Tielrooij, K. J., Koopens, F. H. L. \& Levitov, L. S. Photoexcited carrier dynamics and impact-excitation cascade in graphene. Phys. Rev. B 87, 155429 (2013).

28. Marciniak, A. et al. Electron correlation driven non-adiabatic relaxation in molecules excited by an ultrashort extreme ultraviolet pulse. Nat. Commun. 10, 337 (2019)

29. Marciniak, A. et al. XUV excitation followed by ultrafast non-adiabatic relaxation in PAH molecules as a femto-astrochemistry experiment. Nat. Commun. 6, 7909 (2015).

30. Marciniak, A. et al. Ultrafast nonadiabatic cascade and subsequent photofragmentation of extreme ultraviolet excited caffeine molecule. J. Phys. Chem. Lett. 9, 6927-6933 (2018).

31. Tielens, A. G. G. M. The molecular universe. Rev. Mod. Phys. 85, 1021-1081 (2013).

Publisher's note Springer Nature remains neutral with regard to jurisdictional claims in published maps and institutional affiliations.

(๑) The Author(s), under exclusive licence to Springer Nature Limited 2020 


\section{Methods}

Experimental set-up. The experimental set-up is presented in Supplementary Fig. 1 (Supplementary Information). In this set-up, the XUV-IR interferometer is seeded by a 25 -fs, 2 -mJ, carrier-envelope-phase-stable Ti:sapphire laser, operating at a repetition rate of $5 \mathrm{kHz}$. The beam is split into two equal parts using a 50:50 beamsplitter. The first part is focused (using an $f=30 \mathrm{~cm}$ lens) into a 5 - $\mathrm{mm}$ gas cell filled with rare gas to generate XUV photons through the high-order harmonic generation process. The beam is then reflected on a $\mathrm{Nb}_{2} \mathrm{O}_{5}$-coated mirror to remove most of the remaining infrared light, and is focused with a toroidal mirror towards the molecular jet inside the velocity map imaging (VMI) spectrometer. A 200-nm aluminium filter is inserted in its path to completely remove harmonics below harmonic 13 (that is, the photon energy of the transmitted XUV beam is above $20 \mathrm{eV}$ ), and the XUV spectrum can be measured using a movable grating. The second part of the interferometer is used as the probe arm. Its power can be controlled using a half-wave plate and a polarizer, and is delayed with attosecond precision using a pair of wedges. It is then focused with a $f=1 \mathrm{~m}$ lens and recombined with the XUV pump beam using a motorized holey mirror, enabling spatial overlap of both beams at the focal point. The pump-probe experiment was performed in the interaction region of a VMI/TOF spectrometer. There, gas-phase molecules are injected via an oven filled with the corresponding molecule (in powder), and heated for sublimation of the sample at $80^{\circ} \mathrm{C}$ (diamantane, $\mathrm{C}_{14} \mathrm{H}_{20}$ ), $90^{\circ} \mathrm{C}$ (anthracene, $\mathrm{C}_{14} \mathrm{H}_{10}$ ), $120^{\circ} \mathrm{C}$ (pyrene, $\mathrm{C}_{16} \mathrm{H}_{10}$ ), $210^{\circ} \mathrm{C}$ (tetracene, $\mathrm{C}_{18} \mathrm{H}_{12}$ ), $240^{\circ} \mathrm{C}$ (coronene, $\mathrm{C}_{24} \mathrm{H}_{12}$ ), $290^{\circ} \mathrm{C}$ (pentacene, $\mathrm{C}_{22} \mathrm{H}_{14}$ ), $450^{\circ} \mathrm{C}\left(\mathrm{HBC}, \mathrm{C}_{42} \mathrm{H}_{18}\right.$ ) and $530^{\circ} \mathrm{C}\left(\mathrm{C}_{60}\right)$. Naphthalene $\left(\mathrm{C}_{10} \mathrm{H}_{8}\right)$ and adamantane $\left(\mathrm{C}_{10} \mathrm{H}_{16}\right)$ did not need to be heated due to their high vapour pressure. Delay-dependent TOF spectra were recorded using this configuration. For the largest species, special care was taken to maintain good mass resolution in the TOF spectra by adopting a long field-free drift tube configuration.

Fitting procedure. Following time-dependent measurements, the dication yield was integrated and subtracted from the pump- and probe-only signals, defined as the two-colour signal. This is then fitted to a standard lifetime function according to a least-square fitting procedure:

$$
\begin{aligned}
\Delta S_{2+}(t)= & S_{2+}(t)-S_{\mathrm{XUV}}=\exp \left(-4 \ln (2)\left(\frac{t}{\tau_{\mathrm{Xco}}}\right)^{2}\right) \otimes \\
& {\left[\theta\left(t-t_{0}\right)\left(A_{\text {decay }} \exp \left(-\frac{t-t_{0}}{\tau_{\text {decay }}}\right)+A_{\text {step }}\right)\right] }
\end{aligned}
$$

where $t$ is the XUV-IR delay, $\tau_{\mathrm{Xco}}$ is the experimental XUV-IR cross-correlation duration, $t_{0}$ is delay 0 and $\tau_{\text {decay }}$ the extracted lifetime from the fit.

For each independent measurement, we evaluated the error bars as the standard deviation of the least-square fit. Overall, the cross-correlation duration extracted from these fits is $35 \mathrm{fs}$, imposed by the pulse duration of both infrared and XUV pulses.

Electronic structure calculations. The ionization spectra of the different molecules were computed using the third-order non-Dyson algebraic diagrammatic construction methodology (nD-ADC(3)), with the cc-pVDZ basis set. At this level of theory, all two-hole-one-particle ( $2 \mathrm{~h} 1 \mathrm{p})$ configurations contributing to each state are included. Each line in the spectrum corresponds to a cationic eigenstate. The length of the line, or the spectral intensity, is given by the weight of all 1-hole configurations contributing to the corresponding state, or its monoelectronic part, which can be shown to be related to the partial-channel ionization cross-section. Because all the states are normalized to 1 , the 'invisible' part of the states in the spectrum reflects the contribution of the $2 \mathrm{~h} 1 \mathrm{p}$ configurations, or the multielectronic part of the state. We see that, due to the electron correlation, the $2 \mathrm{~h} 1 \mathrm{p}$ contribution becomes very important after $\sim 15 \mathrm{eV}$ together with an increase in the number of states, whose decreased 1-hole contribution make them less visible in the ADC spectrum. The DOS, extracted from the ADC spectrum, thus becomes a complementary representation of the electronic structure.

\section{Data availability}

Data that support the plots within this paper and other findings of this study are available from the corresponding author upon reasonable request. Source data are provided with this paper.

\section{Code availability}

The codes that support the findings of this study are available from the corresponding authors upon reasonable request.

\section{Acknowledgements}

We thank C. Dujardin and L.S. Cederbaum for fruitful discussions. We acknowledge financial support from CNRS, ANR Circé (ANR-16-CE30-0012). V.D. acknowledges financial support from the DFG through the QUTIF priority programme. A.I.K. thanks US ARO for financial support under grant no. W911NF-14-1-0383. Studies of interstellar PAHs at Leiden Observatory are supported through a grant by the Netherlands Organisation for Scientific Research (NWO) as part of the Dutch Astrochemistry Network and through the Spinozapremie.

\section{Author contributions}

M.H., A.G.G.M.T. and F.L. conceived the project. M.H., P.C.N., V.L., A.B., A.S., G.K., R.B., E.C. and F.L. conducted the experiments. M.H., V.L. and A.B. performed the data analysis. M.H., V.D., A.I.K. and F.L. conceived the theoretical model. V.D. and A.I.K. performed the calculations. M.H. and F.L. wrote the manuscript, with inputs from all the authors. F.L. led the project.

\section{Competing interests}

The authors declare no competing interests.

\section{Additional information}

Supplementary information is available for this paper at https://doi.org/10.1038/ s41567-020-01073-3.

Correspondence and requests for materials should be addressed to F.L.

Peer review information Nature Physics thanks Laura Cattaneo, Mette Gaarde and the other, anonymous, reviewer(s) for their contribution to the peer review of this work.

Reprints and permissions information is available at www.nature.com/reprints. 